\title{
-Quimiluminescência:
}

\section{Princípio e Aplicações}

\section{- Chemiluminescence: principle and applications}

\section{- Quimioluminiscencia: Principio y Aplicaciones}

* Luciana Del Rio Pinoti Ciarlini ${ }^{1}$ - CRMV-SP - no 7329

Paulo César Ciarlini' ${ }^{2}$ - CRMV-SP - n ${ }^{0} \mathbf{6 7 6 7}$

Francisco Leydson Formiga Feitosa ${ }^{3}$ - CRMV-SP - no 5106

Professor Assistente Doutor de Radiologia Veterinária - Unesp/Araçatuba/SP.

Professor Assistente Doutor de Laboratório Clínico Veterinário - Unesp/Araçatuba/SP.

Professor Assistente Doutor de Semiologia Clínica - Unesp/Araçatuba/SP.

\section{RESUMO}

Diversas reações em sistemas biológicos emitem luz, fenômeno chamado quimiluminescência. A quimiluminescência pode ser explicada pelo fato de, na oxidação de certas moléculas, formar-se produtos em um estado eletrônico excitado que emitem luz. Tal emissão de luz em sistemas biológicos está associada a processos como a lipoperoxidação, fagocitose, ciclo redox, etc. O presente trabalho objetiva abordar as principais características da quimiluminescência e suas aplicações, uma vez que esta metodologia vem substituindo com sucesso as técnicas tradicionais, entre elas o radioimunoensaio.

Palavras-chave: Quimiluminescência. Aplicações. Princípios.

\section{Introdução e Revisão da Literatura História da quimiluminescência}

A primeira descrição de bioluminescência foi encontrada no Livro de Odes, um dos treze clássicos datados de 1500-1000 a.C. Escrituras de civilizações antigas contêm referências e dados mitológicos sobre animais luminosos como o vagalume e sobre fenômenos como o corpo brilhante e a floresta incandescente, sendo os gregos e os romanos os primeiros a descrever-lhes as características. Pliny (1855) é citado por ter descrito vários organismos luminosos como a medusa (Pelagia noctiluca), o peixe lanterna (Lucema piscis), o molusco luminoso (Pholas dactylus), o vagalume e os fungos luminosos. Os vagalumes eram chamados pelos gregos de lamparinas por brilharem à noite como "faíscas de fogo". 
CIARLINI, L. D. R. P. CIARLINI, P. C., FEITOSA, F. L. F. Quimiluminescência: Principio e Aplicações / Chemiluminescence: principle and applications Quimioluminiscencia. Principio y Aplicaciones Rev, educ. contin. CRMV-SP / Continuous Education Journal CRMV-SP, São Paulo, volume 5, fasciculo 2, p. 181 - $187,2002$.

Faria-Oliveira et al. (1995) relatam que as observações e descobertas históricas, como a pedra da Bologna, que absorvia a luz do sol e a reemetia no escuro, a incandescência, a luminescência, a luz como partícula, a luz como onda, a fluorescência, a fosforescência, as leis de radiação eletromagnética, entre outras, levaram Wiedmann, em 1888, a estabelecer o nome de quimiluminescência 'a emissão de luz que ocorre como resultado de um processo químico.

Segundo Campbell (1988), o estado da quimiluminescência fornece-nos uma oportunidade ideal para odesenvolvimento da ciência natural como um todo.

\section{Características da quimiluminescência}

A quimiluminescência pode ocorrer em gases, líqüidos e nas interfaces entre gás/líqüido e gás/sólido. Teoricamente, a quimiluminescência pode ocorrer em sólidos, mas poucos trabalhos foram realizados neste campo (FARIA-OLIVEIRA et al., 1995).

Segundo Campbell (1988), há quatro fatores que caracterizam uma reação quimiluminescente, em particular:

1- o espectro da emissão de luz, em particular, se é ou não visível;

2- o estado em que a reação quimiluminescente ocorre, num gás, num líqüido ou numa das interfaces;

3- se a reação quimiluminescente é orgânica ou inorgânica;

4- a existência ou a necessidade de um receptor da energia do produto excitado formado na reação quimiluminescente, constituindo-se na espécie emissiva final, isto é, a transferência de energia ou quimiluminescência sensibilizada.

Segundo Weeks (1997), a eficiência da emissão de luz de uma molécula quimiluminescente é expressa como o rendimento quântico da quimiluminescência $\left(\mathrm{f}_{\mathrm{Cl}}\right)$, o qual descreve o número total de fótons emitidos / número de moléculas reagentes:

$\mathrm{f}_{\mathrm{CL}=} \mathrm{f}_{\mathrm{C}}+\mathrm{f}_{\mathrm{E}}+\mathrm{f}_{\mathrm{F}}$, onde:

$\mathrm{f}_{\mathrm{C}}=$ rendimento químico

$\mathrm{f}_{\mathrm{E}}=$ rendimento de moléculas em estado eletronicamente excitado

\section{$\mathrm{f}_{\mathrm{F}}=$ rendimento quântico da fluorescência}

\section{Sistema Immulite}

Existem vários métodos analíticos que utilizam o princípio da quimiluminescência, porém, o método imunoenzimático amplificado é, atualmente, o mais utilizado na rotina laboratorial. No Brasil, como no exteri- or, este método de análise de quimiluminescência é comercialmente conhecido como Immulite ${ }^{\circledR}$ (DPC Diagnostic Products Corporation, Los Angeles, USA) (Figuras 1, 2 e 3).

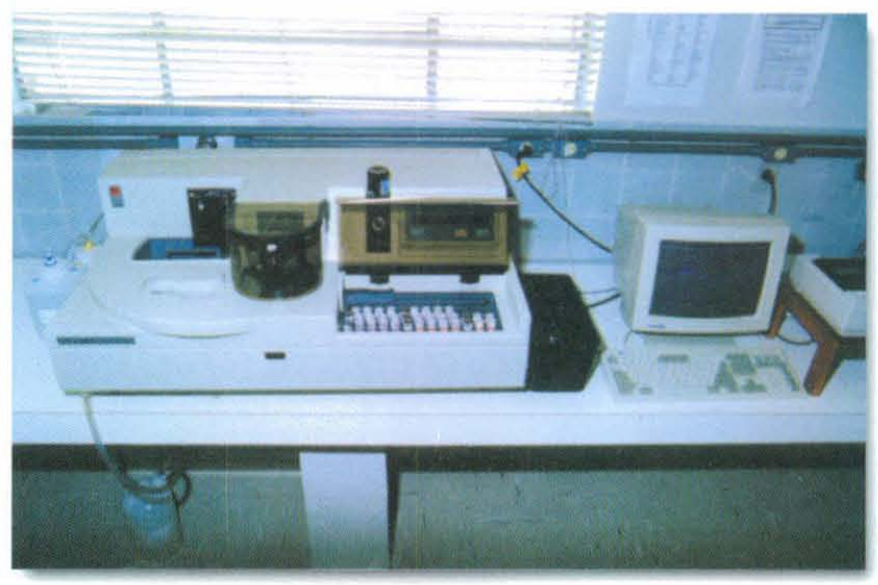

Figura 1 - Foto do aparelho Immulite ${ }^{\infty}$ em funcionamento.

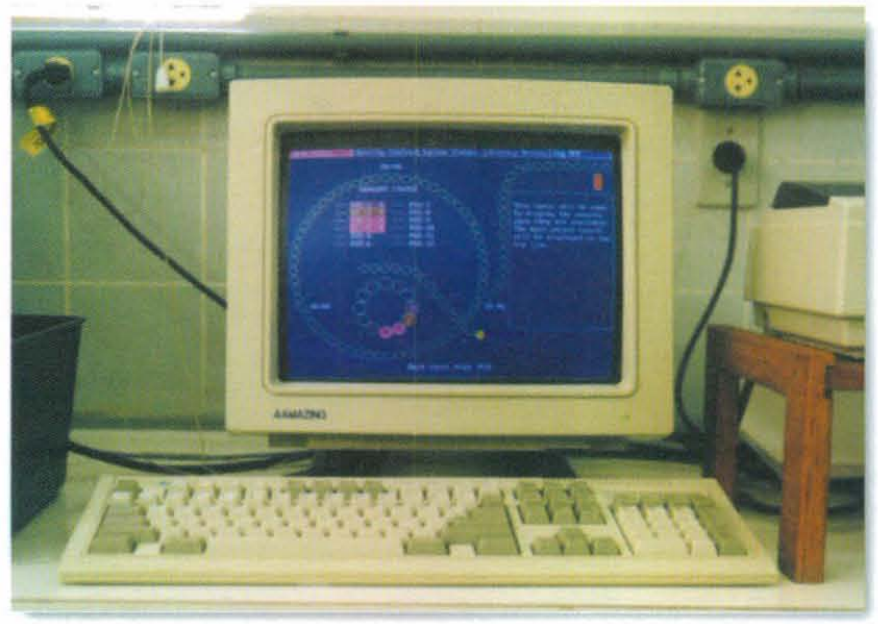

Figura 2 - Tela do computador durante funcionamento do Immulite ${ }^{5}$ demonstrando quais os hormônios que estão sendo dosados.

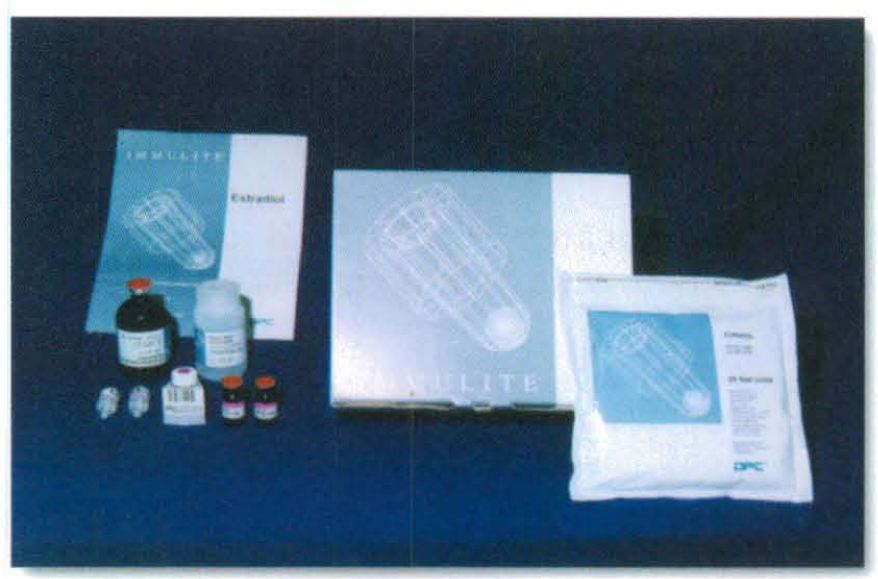

Figura 3 - Partes integrantes do "Kit" comercial para dosagem hormonal. 


\section{Princípio do Sistema Immulite ${ }^{\circledR}$ (Figura 4)}

I- Unidade teste (Figura 5): pérola revestida com anticorpo policlonal específico para o hormônio a ser dosado. Adiciona-se o soro do paciente e a fosfatase alcalina conjugada ao hormônio a ser dosado. Incubase, durante 30 minutos, a $37^{\circ} \mathrm{C}$, sob agitação intermitente.

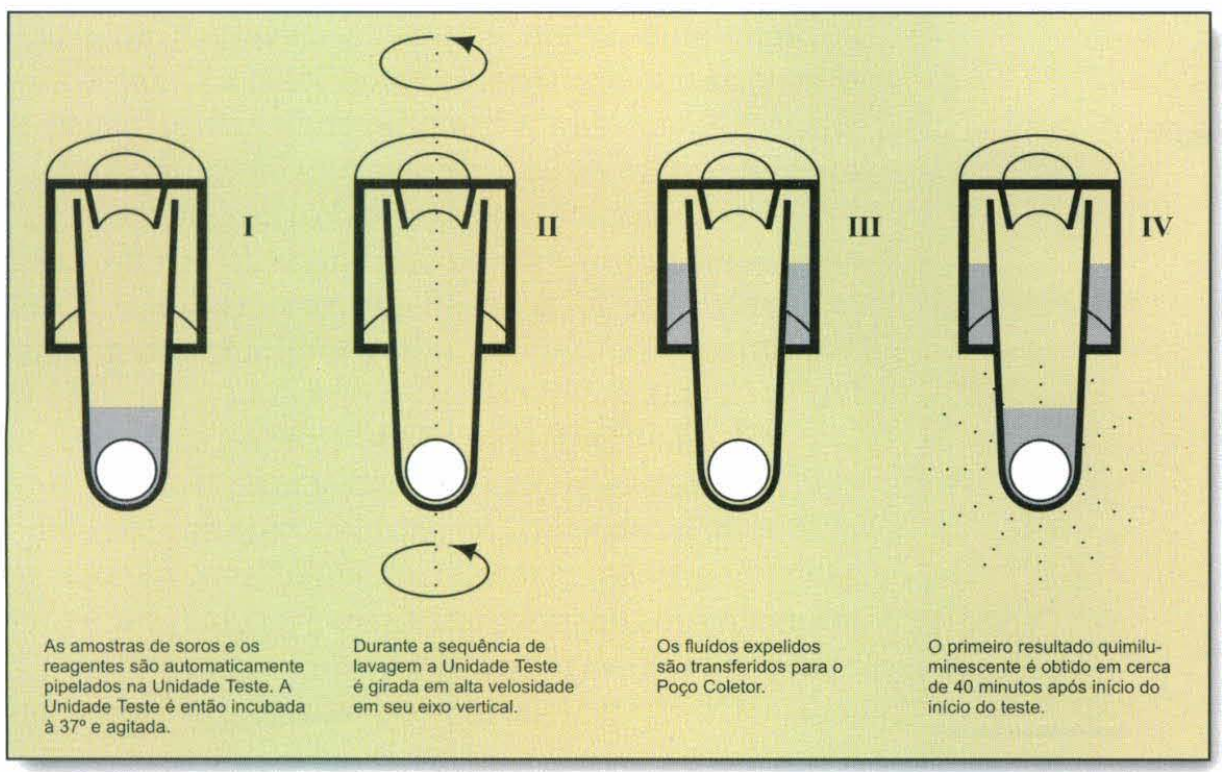

Figura 4 - Principio do sistema Immulite

II- Ocorre a competição entre o hormônio a ser dosado e a enzima marcada com o hormônio, por um número limitado de sítios ligantes do anticorpo da pérola.

III- A enzima conjugada não-ligada é removida por centrifugação para a parte coaxial da unidade teste.

IV-Adiciona-se o substrato quimiluminescente

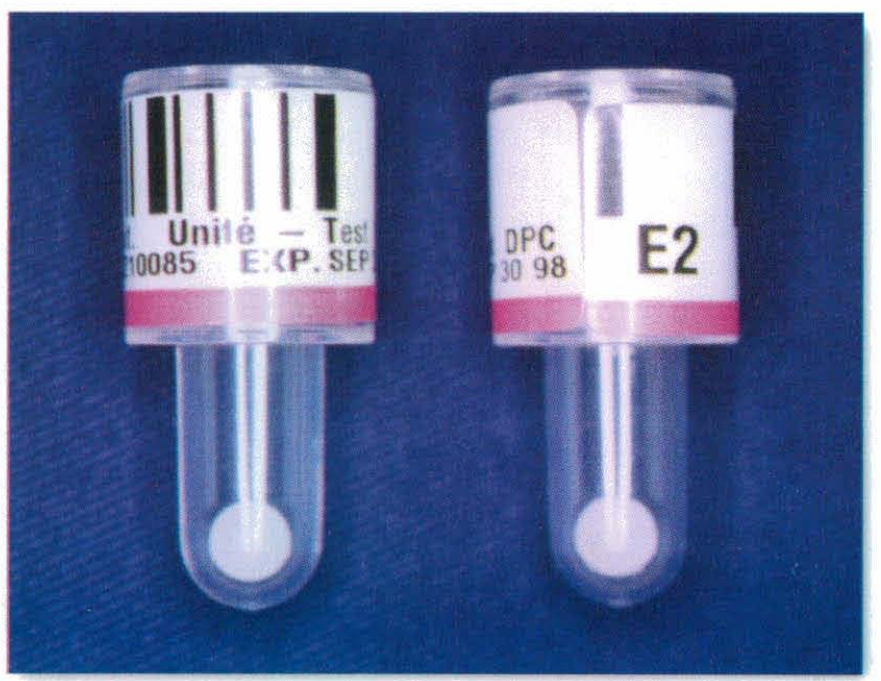

Figura 5 - Detalhe da unidade teste do sistema Immulite ${ }^{\text {b }}$
- éster fosfato de adamantil dioxetano (Figura 6), incuba-se por 10 minutos e realiza-se a leitura no luminômetro.

\section{Aplicações da quimiluminescência na Medici- na Humana e na Veterinária}

\section{Aplicações rotineiras da quimiluminescência}

No comércio estão disponíveis mais de 300 ensaios de quimiluminescência úteis para o diagnóstico de processos alérgicos, neoplásicos e de doenças infecciosas, assim como ensaios destinados à avaliação endócrina, à determinação de citocinas e ao monitoramento de drogas, dentre outras.

Diversos estudos têm demonstrado a eficiência dos ensaios de quimiluminescência, quando comparada a outras metodologias: Armbruster, Jirinzu, Williams (1988), Bigos, MaClean, Butler (1988), Bonini et al. (1988) e Martin, Layte, Aslam (1988a), para Tiroxina; Salinas, Famulare, Worthy (1988), para hormônio paratiroidiano; Kohen, De Boever, Kim (1986), para estradiol e progesterona; Martin, Layte, Aslam (1988a), para cortisol; Armbruster, Jirinzu, Williams (1988) e Barlow et al. (1988), para ferritina.

Entretanto, para a Medicina Veterinária, até o momento, o único ensaio de quimiluminescência disponível comercialmente é o do TSH para a espécie canina. A semelhança do que ocorre com a metodologia do RIA, alguns testes de quimiluminescência disponíveis no comércio podem potencialmente ser utilizados na Medicina Veterinária. Para tal, há necessidade de se fazer mais estudos comparando os ensaios de quimiluminescência com outras metodologias disponíveis.

Atento ao potencial uso da quimiluminescência na Medicina Veterinária, têm sido divulgados alguns resultados iniciais que demonstram a eficiência do sistema Immuliteâ: o resultado da comparação da técnica do radioimunoensaio (RIA) com a técnica da quimiluminescência pelo sistema Immulite ${ }^{\circledR}$. (QL), para dosagem sérica de tiroxina total na espécie felina $(n=57)$, apresentou um alto coeficiente de correlação $(\mathrm{r}=0,959)$; já para tiroxina livre, também na espécie felina $(\mathrm{n}=51)$, 
CIARLINI, L. D. R. P.; CIARLINI, P. C.; FEITOSA, F. L. F. Quimiluminescência: Principio e Aplicações / Chemiluminescence: principle and applications Q Ouimioluminiscencia Principio y Aplicaciones Rev. educ. contin. CRMV-SP/Continuous Education Journal CRMV-SP, São Paulo, volume 5, fasciculo $2, p$. 181 - 187, 2002.

o coeficiente de correlação foi de 0,981 . Para a determinação de digoxina na espécie canina $(n=35)$, o coeficiente de correlação foi de 0,929 ; enquanto para a progesterona em cães $(n=55)$ o "r" foi de 0,921 .

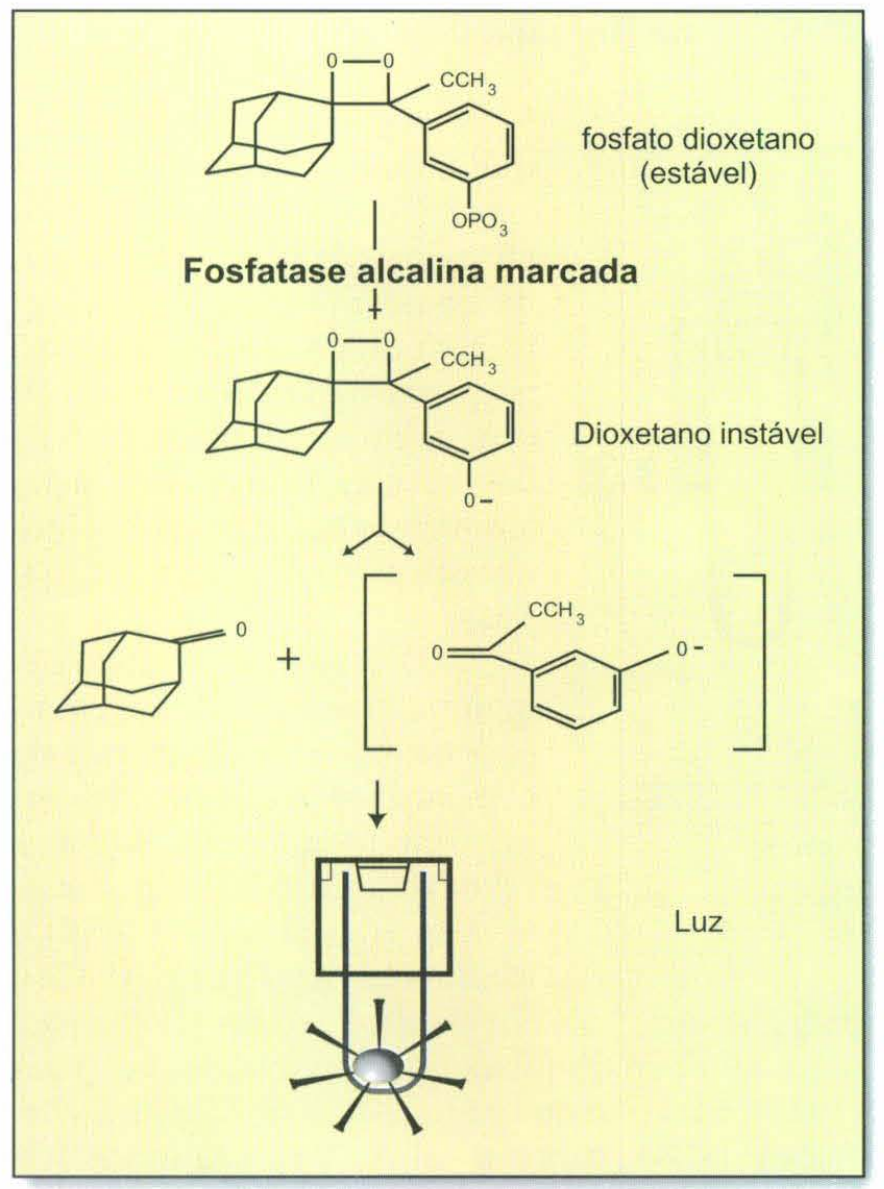

Figura 6 - Substrato quimiluminescente do sistema Immulite I $^{\circledast 2}$

\section{Outras aplicações da quimiluminescência}

Embora não utilizados em laboratórios de rotina, tanto na Medicina Humana como na Veterinária, vários ensaios de quimiluminescência foram desenvolvidos para avaliar o metabolismo celular (ALLEN; STJERNHOLM; STEELE, 1972). Van Dyke e Van Dyke (1986) descreveram um sistema quimiluminescente celular que pode ser usado para quantificar a emissão de luz dos neutrófilos humanos e, portanto, pode ser utilizado para detecção de deficiências genéticas, para estudos de doenças inflamatórias (artrite, asma induzida por exercício e alergia induzida por pólen), para detectar infecção (por bactéria, vírus, micoplasma e leveduras), doenças degenerativas (doença granulomatosa crônica e deficiência de mieloperoxidase) e câncer.

Esta quimiluminescência pode ser mensurada por espectrofotometria e tem sido utilizada no estudo da função bactericida oxigênio-dependente dos fagócitos, no efeito de opsoninas, de vários fatores estimuladores da "explosão respiratória", deficiências genéticas da função neutrofílica e aumento ou supressão da função dos neutrófilos por vários agentes farmacológicos (DULIN; PAAPE; NICKERSON, 1988).

Já Fonseca et al. (1994) descreveram um método quimiluminescente simples e sensível para a caracterização da mieloperoxidase intracelular, útil para a diferenciação entre blastos comprometidos com a linhagem mielóide e linfóide de pacientes com leucemia aguda.

Um imunoensaio enzimático quimiluminescente, com o princípio "sanduíche" usando o marcador oxidase-glicose, foi desenvolvido para quantificar o fator estimulador de colônia nos granulócitos humanos (AOYGAI et al., 1995).

Kohen, De Boever, Kim (1986) desenvolveram um imunoensaio quimiluminescente que permite monitorar a concentração plasmática de drogas, como a digoxina, obtendo resultados similares ao método do imunoensaio com polarização fluorescente, que é rotineiramente utilizado pelos laboratórios clínicos.

Objetivando avaliar o metabolismo de oxi-redução Kamidate e Watanabe (1996) desenvolveram um método quimiluminescente para a determinação de glutation, mensurado indiretamente pela quantidade de peróxido de hidrogênio formado durante a oxidação do glutation.

\section{Radioimunoensaio(RIA)X Quimiluminescência(QL)}

\section{Desvantagens do RIA}

O radioimunoensaio (RIA) é a técnica mais prática e apropriada para realizar as dosagens dos hormônios esteróides e é extensamente utilizada nos laboratórios clínicos e farmacológicos. Entretanto, o grande sucesso e a ampla utilização do RIA está elevando a incidência de sérios problemas, entre eles: 1) conservação e estabilidade dos componentes marcados radioativamente; 2 ) alto custo do material radioativo; 3) perigo à saúde como resultado da exposição e manuseio dos hormônios marcados radioativamente (KOHEN; DE BOEVER; KIM, 1986).

\section{Desvantagens da Quimiluminescência}

A técnica da quimiluminescência foi bem descrita para hormônios esteróides, protéicos e outros componentes biológicos. A principal diferença entre esta técnica e a técnica do radioimunoensaio (RIA) é a 
mensuração do traçador. A mensuração do traçador quimiluminescente é mais difícil do que a mensuração da radioatividade por três razões: 1 ) o decaimento rápido da emissão de luz é necessário para que se dispare tais reações (oxidação), anterior à ação dos fotodetectores, enquanto os radioisótopos espontaneamente emitem radiações b ou g; 2) o sinal analítico obtido pelo traçador quimiluminescente sofre mais variação com o tempo quando comparado ao marcador radioativo, que emite um sinal constante, sendo, portanto, mais facilmente identificável; 3) o sinal da reação quimiluminescente não informa exatamente o número de fótons emitidos e, conseqüentemente, o número de moléculas reacionais (PAZZAGLI; SERIO, 1986).

\section{Vantagens da Quimiluminescência}

A quimiluminescência, comparada ao radioimunoensaio com $\mathrm{I}^{125}$, fornece ao usuário algumas vantagens: 1) não utiliza material radioativo, não havendo, desta maneira, os problemas inerentes ao manuseio de substâncias radioativas, como: proteção radiológica, descarte apropriado, monitorização do pessoal, etc; 2) possui prazos maiores de validade do que as metodologias tradicionais de radioimunoensaio; 3) automação: método automatizado, em que não há manuseio de amostras e reagentes, evitando-se, assim, os tradicionais erros de pipetagem e trocas de reagentes; com isso, é possível também propiciar técnicas com resultados muito mais apurados do ponto de vista do coeficiente de variação intra-ensaio e, além disso, diminui o risco de acidentes com materiais biológicos ou reagentes, oferecendo maior segurança ao operador; 4) em virtude da alta performance de automação do aparelho, as transferências de líqüidos e pipetagens são feitas de maneira precisa, conferindo, assim, segurança para a execução dos testes em uniplicata 5) possibilita a execução de vários testes diferentes em uma mesma amostra de uma única vez, oferecendo maior rapidez para a obtenção dos resultados (IMMULITE, 1996).

\section{Vantagens do Radioimunoensaio}

Embora utilize marcadores radioativos, o custo quando comparado à quimiluminescência é muito menor.

\section{Conclusões}

Nos últimos anos ocorreu um grande desenvolvimento de metodologias de ensaios analíticos baseadas no princípio da quimiluminescência, cuja eficiência tem sido comprovada por inúmeros estudos. A importância da aplicação desta metodologia em diferentes áreas da Medicina Humana e Veterinária é indiscutível, particularmente por ser um recurso analítico ecologicamente mais correto, não baseada em radioisótopos e, portanto, dentro dos preceitos modernos de biossegurança.

\section{Agradecimentos}

À Dr ${ }^{\mathrm{a}}$ Adriana Polachini do Valle, responsável pelo Laboratório de Endocrinologia e Metabolismo do Hospital das Clínicas - UNESP - Botucatu

\section{SUMMARY}

Light is emitted by several reactions occurring in biological systems, a phenomenon known as chemiluminescence. It is associated with oxidative reactions that form products in an excited electronic state, reactions such as lipoperoxidation, phagocytosis, redox cycle, etc. Based on the existing literature, this article presents the main characteristics of chemiluminescence and its applications. This method has rendered several traditional techniques, such as radioimmunoassay, obsolete.

Key words: Chemiluminescence. Applications. Principle. 


\section{RESUMEN}

Diversas reacciones en sistemas biológicos emiten luz, fenómeno llamado de quimioluminiscencia. La quimioluminiscencia puede explicarse por el hecho de que en la oxidación de ciertas moléculas se forman productos en un estado electrónico excitado que emiten luz. Dicha emisión de luz en sistemas biológicos está asociada a procesos tales como lipoperoxidación, fagocitosis, ciclo redox, etc. El presente trabajo tiene como objeto abordar las principales características de la quimioluminiscencia y sus aplicaciones, ya que esta metodología viene reemplazando exitosamente las técnicas tradicionales, entre ellas el radioinmunoensayo.

Palabras clave: Quimioluminiscencia. Aplicaciones. Principios.

\section{REFERÊNCIAS}

ALLEN, R. C; STJERNHOLM, R. L.; STEELE, R. H. Evidence for the generation of an electronic excitation state(s) in human polymorphonuclear leukocytes and its participation in bactericidal activity. Biochem. Biophys. Res. Com., v. 47, n. 4, p. 679684, 1972.

AOYGAI, S. et al. Chemiluminescence sandwich enzyme immunoassay for determination of human granulocyte colony stimulating factor (G-CSF). J. Biolumin. Chemilumin., v. 10, n. 6, p. 345-351, 1995.

ARMBRUSTER, D. A.; JIRINZU, D. C.; WILLIAMS, J. V. Enhanced luminescence immunoassays for free thyroxine (FT4) and ferritin. Clin. Chem., v. 34, p. 1153, 1988. Abstract.

BARLOW, E. et al. A chemiluminescent immunoassay for measurement of ferritin. Clin. Chem., v. 34, p. 1162, 1988. Abstract.

BIGOS, S. T.; MACLEAN, J.; BUTLER, B. Comparison of free thyroxine measurement by chemiluminescence and equilibrium dialysis. Clin. Chem., v. 34, p. 1214, 1988. Abstract.

BONINI, P. A. et al. Free thyroxin estimation by enhanced luminescent immunometry. Clin. Chem., v. 34, p. 1207, 1988. Abstract.

CAMPBELL, A. K. Chemiluminescence: principles and applications in biology and medicine. England: VCH \& Ellis Horwood, 1988. p. 607.
DULIN, A. M.; PAAPE, M. J.; NICKERSON, S. C. Comparison of phagocytosis and chemiluminescence by blood and mammary gland neutrophils from multiparous and nulliparous cows. Am. J. Vet. Res., v. 49, p. 172-177, 1988.

FARIA-OLIVEIRA, O. M. M. et al. Quimiluminescência: Aspectos químicos e físicos. Rev. Ciênc. Farm., v. 16, p. 9-22, 1995.

FERRY, J. D. et al. Magic lite - Evaluation of FT4, T4, T3, T3 uptake, Free thyroxine index (FTI) and TSH by chemiluminescence vs ${ }^{125}$ method. Clin. Chem., v. 34, p. 1182, 1988.

FONSECA, L. M. et al. Quimiluminescência em células blásticas leucêmicas e sua aplicação no diagnóstico diferencial de leucemias agudas. Rev. Bras. Anál. Clín., v. 26, p. 2-6, 1994.

HARVEY, E. N. A history of luminescence. Philadelphia: American Philadelphical Society, 1957. p. 300.

HASTINGS, J. W. Biological diversity, chemical mechanism, and evolutionary origins of bioluminescence systems. J. Mol. Evol. (review), v. 19, p. 329-331, 1983.

IMMULITE: manual de operação. São Paulo: DPC MED LAB, 1995. $125 \mathrm{p}$.

IMMULITE: veterinary methods comparisons. Los Angeles: DPC, $1996.25 \mathrm{p}$. 
JOHN, R. et al. Evaluation of a new strategy for detection of thyroid dysfunction in the routine laboratory. Clin. Chem., v. 34, p. 1110-1114, 1988.

KAMIDATE, T.; WATANABE, H. Peroxidase-catalysed luminol chemiluminescence method for the determination of glutathione. Talanta, v. 43, p. 1733-1738, 1996.

KOHEN, F.; DE BOEVER, J.; KIM, J. B. Surface Chemiluminescent Immunoassays of Steroids. Methods Enzymol., v. 133, p. 387, 1986.

MARTIN, J. K.; LAYTE, K.; ASLAM, M. Enhanced luminescence immunoassay for free T4. Clin. Chem., v. 34, p.1216, 1988a. Abstract.

MARTIN, J. K. et al. Measurement of cortisol with a non-radioactive immunoassay based on enhanced luminescence. Clin. Chem., v. 34, p. 1217, 1988b. Abstract.
MEDEIROS, M. H. G.; BAPTISTA, R. C.; DI MASCIO, P. Quimiluminescência fraca em sistemas biológicos. Rev. Ciênc. Farm., v. 16, p. 23-35, 1995.

PAZZAGLI, M.; SERIO, M. On-line computer analysis of the kinetics of chemiluminescent reactions: application to luminescent immunoassays. Methods Enzymol., v. 133, p. 420-435, 1986.

SALINAS, R.; FAMULARE, A. J.; WORTHY, T. E. Evaluation of a chemiluminometric assay for intact parathyroid hormone (Intact PTH). Clin. Chem., v. 34, p. 1157, 1988. Abstract.

VAN DYKE, K., VAN DYKE, C. Cellular chemiluminescence associated with disease states. Methods Enzymol., v. 133, p. 493-494, 1986.

WEEKS, I. Chemiluminescence Immunoassay. In: PRICE, C. P. Principles and practice of immunoassay. $2^{\text {th }}$ ed. Londres: MacMilan, 1997. chapter 17, p. 427-442. 